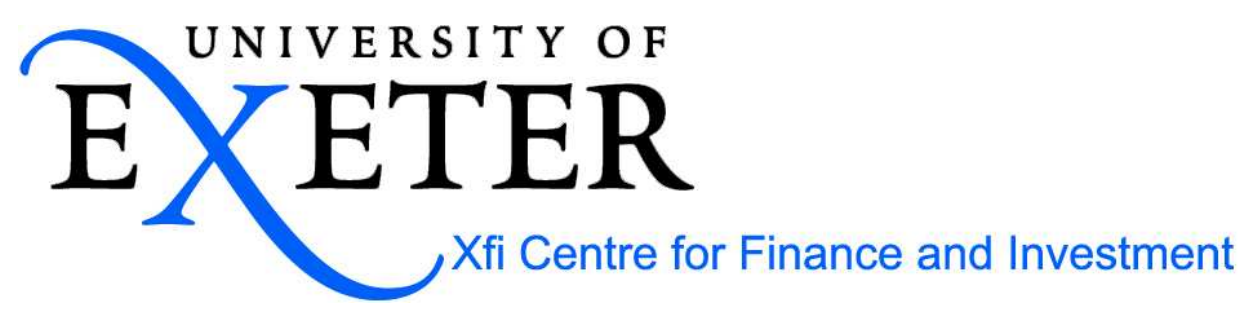

\title{
Return and Volatility Spillovers Between Large and Small Stocks in the UK
}

\author{
Richard D. F. Harris \\ Xfi Centre for Finance and Investment \\ University of Exeter, $U K$ \\ Anirut Pisedtasalasai \\ Department of Accounting, Finance and Information Systems \\ University of Canterbury, New Zealand
}

August 2005

Paper Number: 06/09

\begin{abstract}
This paper investigates return and volatility spillover effects between the FTSE 100, FTSE 250 and FTSE Small Cap equity indices using the multivariate GARCH framework. We find that return and volatility transmission mechanisms between large and small stocks in the UK are asymmetric. In particular, there are significant spillover effects in both returns and volatility from the portfolios of larger stocks to the portfolios of smaller stocks. For volatility, there is also evidence of limited feedback from the portfolios of smaller stocks to the portfolios of larger stocks, although sub-period analysis suggests that this is to some extent period-specific. Simulation evidence shows that non-synchronous trading potentially explains some, but not all, of the spillover effects in returns, and that it explains none of the spillover effects in volatility. These results are consistent with a market in which information is first incorporated into the prices of large stocks before being impounded into the prices of small stocks.
\end{abstract}

KEYWORDS: Return and volatility spillovers; Multivariate GARCH; FTSE indices. Address for Correspondence: Richard D.F. Harris, Xfi Centre for Finance and Investment, University of Exeter, Exeter EX4 4ST, UK. Email: R.D.F.Harris@exeter.ac.uk. 


\section{Introduction}

Transmission mechanisms between the returns and volatilities of different stocks are important for a number of reasons. Firstly, transmission mechanisms tell us something about market efficiency. In an efficient market, and in the absence of time-varying risk premia, it should not be possible to forecast the returns of one stock using the lagged returns of another stock. The finding that there are spillover effects in returns implies the existence of an exploitable trading strategy and, if trading strategy profits exceed transaction costs, potentially represents evidence against market efficiency. Secondly, transmission mechanisms may be useful for portfolio management, where knowledge of return spillover effects may be useful for asset allocation or stock selection. Thirdly, information about volatility spillover effects may be useful for applications in finance that rely on estimates of conditional volatility, such as option pricing, portfolio optimization, value at risk and hedging.

Many previous studies have documented that the returns of large and small stocks in the US stock market are cross-correlated. ${ }^{1}$ Moreover, a number of these studies show that these cross-correlations are asymmetric: the returns of small stock portfolios tend to be correlated with the lagged returns of large stock portfolios, while the returns of large stock portfolios tend to be uncorrelated with the lagged returns of small stock portfolios. Lo and MacKinlay (1990a, 1990b) rule out non-synchronous trading as an explanation since implausible levels of non-synchronous trading are required to generate the size of the cross-correlations that exist in practice. A number of other explanations have therefore been proposed. Mech (1993) suggests that asymmetry in the cross-correlation between returns on large and small stocks is due to transaction costs, and shows that the speed of price adjustment is associated with the standard deviation of returns and the bid-ask spread. Chan (1993) suggests that differences in signal quality between large and small stocks induce asymmetry in their crosscorrelations. In particular, if the signal quality of large stocks is assumed to be better than that of small stocks, the covariance of the current returns of small stocks with the lagged returns of large stocks is larger than the covariance of the current returns of

\footnotetext{
${ }^{1}$ See, for example, Lo and MacKinlay (1990a, 1990b), Boudoukh, Richardson and Whitelaw (1994), Mech (1993), Badrinath, Kale and Noe (1995), McQueen, Pinegar and Thorley (1996) and Campbell, Lo and MacKinlay (1997, pages 74-78).
} 
large stocks with the lagged returns of small stocks. Some studies (for example, Grinblatt, Titman, and Wermers, 1995; Keim and Madhavan, 1995) argue that asymmetric spillover effects in the returns of large and small stocks are related to asymmetric trading patterns and the behavior of institutional investors.

Conrad, Gultekin and Kaul (1991) show that the same asymmetry that exists in the transmission of short horizon returns between large and small stocks in the US also exists in the transmission of volatility. They find that volatility shocks to large stocks are important for the future volatility of small stocks, but that volatility shocks to smaller stocks have little or no impact on the future volatility of large stocks. As with the results for return spillovers, simulation evidence suggests that the observed spillover effects in volatility are not caused by non-synchronous trading. Conrad Gultekin and Kaul (1991) note that since stock price volatility is directly related to the rate of flow of information to the market (see Ross, 1989), the asymmetry in volatility spillovers between large and small stocks is consistent with a market in which the prices of large stocks respond to new information immediately, but the prices of small stocks respond with a lag. This explanation is supported by McQueen, Pinegar and Thorley (1996), who show that small stocks display a delayed reaction compared to large stocks when news reaches the market. Using longer horizon returns, Hasan and Francis (1998) also find that there are volatility spillovers between small and large stocks in the US, but in contrast with Conrad Gultekin and Kaul (1991), they find that these spillovers are approximately symmetric, acting both from large stocks to small stocks, and from small stocks to large stocks.

This paper investigates the return and volatility transmission mechanisms between large and small stocks in the UK stock market using daily data on the FTSE 100, FTSE 250 and FTSE Small Cap equity indices. We investigate these transmission mechanisms using the constant correlation multivariate GARCH model of Bollerslev (1990). We model the spillover effects by introducing into the mean and variance equation for each index, the lagged shocks to the returns and volatilities of the other two indices. To ensure that our results are robust, we include dummy variables to capture outliers, calendar effects and the asymmetric response of volatility to good and bad news. To further test the robustness of our results, we conduct the analysis using both the full sample and two sub-samples. Our results show that there are strong 
return and volatility transmission mechanisms between small and large stocks in the UK stock market. Furthermore, consistent with the results of other studies for the US, we find that these return spillover effects are asymmetric. In particular, there are very significant return spillovers from the portfolios of large stocks to the portfolios of small stocks. For volatility, there are again positive spillovers from the portfolios of large stocks to the portfolios of small stocks, although there is also evidence of feedback from the portfolios of smaller stocks to the portfolios of larger stocks, and in one case, this feedback effect is negative. However, for the sub-samples, the pattern of volatility spillovers is very similar to that for return spillovers, with only limited positive feedback from small stock portfolios to large stock portfolios.

In order to analyse the effect of non-synchronous trading on our results, we undertake a Monte Carlo simulation experiment in which data are simulated for portfolios of small, medium and large stocks. The individual stocks in the three portfolios are characterised by varying probabilities of non-trading, which are estimated using the empirical non-trading frequencies for the three FTSE indices. The simulation results suggest that non-synchronous trading leads to spillover effects in returns, although the simulated spillover effects are not as large as those that are observed empirically. Similarly, the simulation evidence suggests that non-synchronous trading cannot explain the observed pattern of spillover effects in volatility. We therefore conclude that our empirical results are consistent with a market in which information is first incorporated into the prices of large stocks before being impounded into the prices of small stocks.

The remainder of the paper is organized as follows. The following section describes the data that we use in the study. Section 3 gives details of the empirical methodology. Section 4 reports the results. Section 5 presents the results of the Monte Carlo simulation. Section 6 offers a summary and conclusion.

\section{Data Description}

The empirical analysis uses continuously compounded daily stock returns for the FTSE 100, FTSE 250 and FTSE Small Cap indices from 1 January 1986 to 31 December 2002, obtained from Datastream (a total of 4,435 observations). The FTSE 
100, FTSE 250 and FTSE Small Cap indices are market-weighted indices that account for approximately 85 percent, 12 percent and 3 percent of the FTSE All Share index, respectively, at the end of 2002. We use short horizon returns because we want to analyse spillover effects in both the mean and volatility of the three series, but time series variation in conditional volatility tends to be much weaker for longer horizon returns. For the sub-period analysis, we split the full sample into two equal subperiods. The first sub-sample is from 1 January 1986 to 30 June 1994, while the second sub-sample is from 1 July 1994 to 31 December 2002.

Table 1 reports summary statistics for the three return series for the full sample. Panel A reports the mean, standard error, skewness and excess kurtosis coefficients and the Jarque-Bera statistic to test the null hypothesis that returns are normally distributed. All three series are negatively skewed and highly leptokurtic, and the Jarque-Bera statistic rejects the null hypothesis of normality very strongly. Panel B of Table 1 reports the first four autocorrelation coefficients for returns and squared returns for each index, together with Ljung-Box portmanteau statistics. For all three indices, returns are serially correlated, although the magnitude of the serial correlation decreases with capitalisation. Squared returns are highly serially correlated for all three series, indicating the presence of volatility clustering. In contrast with the pattern of serial correlation in returns, the magnitude of the serial correlation in squared returns increases with capitalisation, implying that $\mathrm{ARCH}$ effects are stronger for large stocks than for small stocks.

[Table 1]

\section{Methodology}

\subsection{Modelling the Returns and Volatilities of the Indices}

As a benchmark, we first model the dynamic properties of the returns and volatilities of the FTSE 100, FTSE 250 and FTSE Small Cap return series without spillover effects. We use the following multivariate AR-GJR-GARCH-M model for the three indices $i, j=1,2,3$ 


$$
\begin{aligned}
R_{i, t}= & \alpha_{i, 0}+\sum_{n=1}^{4} \alpha_{i, n} R_{i, t-n}+\gamma_{i} h_{i i, t}+c_{i, 1} \text { OCT } 87_{t}+c_{i, 2} A \text { SIA } 97_{t} \\
& +c_{i, 3} J A N_{t}+c_{i, 4} M O N_{t}+\varepsilon_{i, t} \\
h_{i i, t}= & \beta_{i, 0}+\beta_{i, 1} h_{i i, t-1}+\beta_{i, 2} \varepsilon_{i, t-1}^{2}+\lambda_{i} I_{i, t-1} \varepsilon_{i, t-1}^{2}+d_{i, 1} O C T 87_{t}+d_{i, 2} A S I A 97_{t} \\
& +d_{i, 3} J A N_{t}+d_{i, 4} M O N_{t} \\
h_{i j, t} & =\rho_{i j}\left(\sqrt{h_{i i, t}} \sqrt{h_{i j, t}}\right)
\end{aligned}
$$

where $R_{i, t}$ is the return of portfolio $i$ in period $t, \varepsilon_{t} \mid \psi_{t-1} \sim N\left(0, H_{t}\right), \psi_{t-1}$ is the set of all information available at time $t-1$ and $H_{t}=\left[h_{i j, t}\right]$ is the conditional covariance matrix. $I_{i, t-1}$ is a dummy variable that is equal to one if the lagged shock to returns, $\varepsilon_{i, t-1}$ is negative, and zero otherwise. OCT87 ${ }_{t}$ is a dummy variable that is equal to one for observations between $19 / 10 / 87$ and $4 / 11 / 87$ and zero otherwise. ASIA97 ${ }_{t}$ is a dummy variables that is equal to one for observations between 23/10/97 and 28/10/97 and zero otherwise. $J A N_{t}$ is a dummy variable that is equal to one for the first week in January and zero otherwise. $M O N_{t}$ is a dummy variable that is equal to one for Mondays and zero otherwise.

The AR(4) specification for the mean equation (1) was chosen on the basis of the Schwartz Bayesian Criterion (SBC) from a general $\operatorname{ARMA}(p, q)$ specification, although the model selected by the Akaike Information Crierion (AIC) led to broadly similar empirical results. We have specified a GARCH-in-mean term, with the conditional variance of each index included as an explanatory variable in the respective mean equation. This is to exploit as much information in estimating expected returns, rather than to impose a particular asset pricing restriction, and is common in the literature. ${ }^{2}$

${ }^{2}$ See, for example, Hamao, Masulis and Ng (1990), Conrad, Gultekin and Kaul (1991), Ng, Chang and Chou (1991) and Theodossiou and Lee (1993). 
The model for the conditional covariance matrix, given by (2) and (3), is based on the constant correlation (CCOR) specification of the multivariate GARCH model of Bollerslev (1990). The model specifies the conditional variance of each index as a univariate GARCH model, with the conditional covariance of any two series $i$ and $j$ determined by the conditional variances, $h_{i i, t}$ and $h_{j j, t}$, and the constant correlation coefficient, $\rho_{i j}$. While there are several multivariate GARCH models to choose from, the CCOR model has the advantage of being parsimonious and hence greatly reduces the computational effort required to estimate the model, generally leading to more reliable parameter estimates. ${ }^{3}$ This is particularly important in the present case owing to the large number of parameters to be estimated. The $\operatorname{GARCH}(1,1)$ specification for the conditional variance in equation (2) was chosen for the sake of parsimony.

In order to ensure the robustness of our results, we modify the CCOR model in several ways. Firstly, it is well documented that volatility responds asymmetrically to good and bad news and, moreover, that volatility spillovers can be significantly understated if this asymmetric effect is ignored. ${ }^{4}$ To capture the asymmetric effect of news on volatility, we use the GJR specification of the multivariate GARCH model and include a dummy variable for negative return shocks (see Glosten, Jagannathan and Runkle, 1993). Secondly, to allow for outliers in the data, we include dummy variables in both the return and volatility equations for periods of extreme market movements. The large negative returns in international equity markets during the week following the stock market crash of 19 October 1987 are well documented. So too is the increase in the conditional volatility of returns. ${ }^{5}$ Following Aggarwal, Inclan and Leal (1999), we include in the full sample and the first sub-sample, a dummy variable for the two weeks following the stock market crash of 1987 . We also allow for the 1997 Asian financial crisis by including in the full sample and the second subsample, a dummy variable for the week that marked the start of the crisis (see Wang, Rui, and Firth, 2002). Lastly, we also include dummy variables in both the mean equation and the variance equation for the well-known January and Monday effects. Many studies have shown that returns are systematically higher during the first week

\footnotetext{
${ }^{3}$ See Engle and Kroner (1995), Kroner and Ng (1998).

${ }^{4}$ See Glosten, Jagannathan and Runkle (1993), Engle and Ng (1993), Bae and Karolyi (1994), Wang, Rui and Firth (2002) and Hung, Lee and So (2003).
} 
of January than in other months of the year, and lower on Mondays than on other days of the week. ${ }^{6}$ A number of studies, including Conrad, Gultekin and Kaul (1991), have also found that there are similar calendar effects in the volatility of returns.

\subsection{Modelling Spillover Effects Between the Indices}

To analyse the return and volatility spillovers between the FTSE 100, FTSE 250 and FTSE Small Cap indices, we modify the AR-GJR-GARCH-M model given by (1)-(3) to include in the mean and variance equations for each index, the lagged shocks to the means and volatilities of the other two indices. Specifically, to capture spillover effects in the mean equation for index $i$, we include the first lag of the returns of each of the indices $i \neq j$. To capture spillover effects in the volatility equation for index $i$, we include the first lag of the squared return shocks of each of the indices $i \neq j$. The model including spillovers for indices $i, j=1,2,3$ is therefore given by

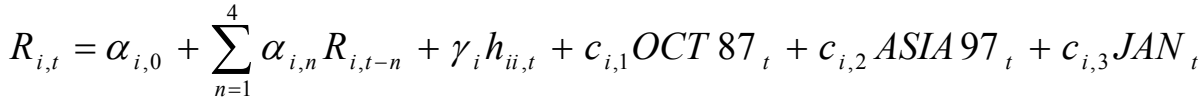

$$
\begin{aligned}
& +c_{i, 4} M_{t}+\sum_{j=1, j \neq i}^{3} w_{i, j} R_{j, t-1}+\varepsilon_{i, t} \\
& h_{i i, t}=\beta_{i, 0}+\beta_{i, 1} h_{i i, t-1}+\beta_{i, 2} \varepsilon_{i, t-1}^{2}+\lambda_{i} I_{i, t-1} \varepsilon_{i, t-1}^{2}+d_{i, 1} \text { OCT } 87_{t}+d_{i, 2} \text { ASIA97 }_{t} \\
& +d_{i, 3} J A N_{t}+d_{i, 4} M O N_{t}+\sum_{j=1, j \neq i}^{3} z_{i, j} \varepsilon_{j, t-1}^{2} \\
& h_{i j, t}=\rho_{i j}\left(\sqrt{h_{i i, t}} \sqrt{h_{i j, t}}\right)
\end{aligned}
$$

The parameter $w_{i, j}$ measures the partial impact on the returns of index $i$ of past return shocks of the two remaining indices, while the parameter $z_{i, j}$ measures the partial impact on the volatility of index $i$ of past volatility shocks to the two remaining indices. We estimate the multivariate GARCH model, both with and without spillover

\footnotetext{
${ }_{6}^{5}$ See Roll (1988), Schwert (1990), Aggarwal, Inclan and Leal (1999).

${ }^{6}$ For evidence of the January effect, see, for example, Tinic and West (1984), Lakonishok and Smidt (1988) and Draper and Paudyal (1997).
} 
effects, by quasi-maximum likelihood with a normal conditional distribution (see Bollerslev and Woolridge, 1992). We use the BFGS algorithm with a convergence criterion of 0.00001 applied to the function value. Robust errors are computed that are valid under non-normality (see White, 1982).

\section{Empirical Results}

\subsection{Multivariate AR(4)-GJR GARCH(1,1)-M Results}

Table 2 reports the estimated parameters of the multivariate AR(4)-GJRGARCH(1,1)-M model for each the three indices, given by equations (1), (2) and (3), together with Ljung-Box test statistics for the standardized residuals (LB(4)) and the squared standardized residuals $\left(\mathrm{LB}^{2}(4)\right)$. For all three indices, the sum of the estimated GARCH parameters, $\beta_{i, 1}+\beta_{i, 2}$, suggests that volatility is stationary but highly persistent. In particular, the half-life of volatility for the FTSE 100, FTSE 250 and FTSE Small Cap indices is 20.88 days, 9.74 days and 17.15 days, respectively. ${ }^{7}$ The GARCH-in-mean coefficient, $\gamma_{i}$, is significantly positive for all three indices, implying that higher volatility is associated with higher expected returns, which is consistent with risk aversion. The coefficient of the asymmetry term, $\lambda_{i}$, is significantly positive for the FTSE 100 index, implying that bad news has a larger impact on the volatility of the FTSE 100 index than good news does. The asymmetry term is also positive for the FTSE 250 and FTSE Small Cap indices, but not statistically significant. As expected, the estimated correlation coefficients, $\rho_{i j}$, among the three indices are positive and highly significant.

There is strong evidence of the effect of the October 1987 crash on the returns and conditional variances of the three indices. In particular, returns are very significantly negative during the crash, and for the FTSE 250 and FTSE Small Cap indices, volatility is significantly higher. The Asian crisis had a significant negative impact on returns all three indices, and a marginally significant positive impact on volatility. The

7 The half-life is computed as $h_{i}=\ln (1 / 2) / \ln \left(\beta_{i, 1}+\beta_{i, 2}+\lambda_{i} / 2\right)$ under the assumption that the return distribution is symmetric. 
January effect is insignificant for returns, but has a positive impact on volatility, which is significant for the FTSE 250 index, and marginally significant for the FTSE 100 and FTSE Small Cap indices. The Monday effect is significantly negative for FTSE 100 and FTSE 250 returns, and marginally significantly negative for FTSE Small Cap returns. For volatility, the Monday dummy is significantly negative for the FTSE 250 index, and negative with marginal significance for the FTSE 100 index.

The $\operatorname{LB}^{2}(4)$ statistics suggest that the multivariate $\operatorname{GARCH}(1,1)$ specification successfully captures the serial correlation in squared returns for each of the three indices. The $\mathrm{LB}(4)$ statistics show that there is significant serial correlation in the residuals for the three indices. However, it will be seen below that this serial correlation is significantly reduced once we include return and volatility spillovers into the model. Alternative ARMA specifications of the mean equation (particularly those selected by the AIC, which generally include longer lags of both the AR and MA components) failed to eliminate this serial correlation.

[Table 2]

Table 3 reports the estimated parameters of the multivariate AR(4)-GJR GARCH(1,1)-M model with spillover effects for each the three indices, given by equations (4), (5) and (6). Comparing Table 2 and Table 3, it can be seen that the introduction of the spillover effects into the model generally has only a small effect on the estimated parameter values for the mean and variance equations of the three indices. Moreover, the introduction of spillover effects significantly reduces the serial correlation in the residuals. The $\mathrm{LB}(4)$ statistic is now insignificant for the FTSE Small Cap Index, and considerably reduced for the FTSE 100 and FTSE 250 indices. Again, alternative ARMA specifications of the mean equation failed to completely eliminate the remaining serial correlation. However, the choice of model for the mean return for these series does not significantly affect the results on mean and volatility spillovers that are reported below, and alters none of the qualitative conclusions. ${ }^{8}$

\footnotetext{
${ }^{8}$ Results obtained using alternative specifications of the mean equation are available from the authors.
} 
Table 3 shows that there are very significant return and volatility spillovers between the FTSE 100, FTSE 250 and FTSE Small Cap indices. Moreover, these spillover effects are highly asymmetric. In particular, there are significant positive spillover effects in returns from the FTSE 100 index to the FTSE 250 and FTSE Small Cap indices. There is also a marginally significant positive spillover from the FTSE 250 index to the FTSE Small Cap index. In contrast, there are no statistically significant spillover effects from the portfolios of smaller stocks to the portfolios of larger stocks. Consistent with the findings of previous studies for the US cited above, we therefore find that there are very significant asymmetric spillover effects from the returns of large stocks to the returns of small stocks.

For conditional volatility, the spillover effects are more complex. We again find that there are significant spillover effects from the FTSE 100 index to both the FTSE 250 index and the FTSE Small Cap index and from the FTSE 250 index to the FTSE Small Cap index. These findings are consistent with those of Conrad, Gultekin and Kaul (1991) for the US. However, we also find that there is evidence of feedback in volatility from smaller stocks to larger stocks, with a significant negative spillover between the FTSE 250 index and the FTSE 100 index, implying that an increase in the volatility of the FTSE 250 index is associated with a subsequent decrease in the volatility of the FTSE 100 index. There is also a positive spillover in volatility from the FTSE Small Cap index to the FTSE 100 index, and a marginally significant positive spillover from the FTSE Small Cap index to the FTSE 250 index. In order to shed more light on the observed spillover patterns, we conduct the same analysis using each of the two sub-samples.

[Table 3]

\subsection{Sub-Period Analysis}

Table 4 presents the results for the first sub-sample, while Table 5 presents the results for the second sub-sample. The pattern of return spillovers for both sub-samples are very similar to those reported for the full sample. We again find that the spillover effects in returns are highly asymmetric in both sub-samples. There are highly significant positive spillover effects in returns from the FTSE 100 index to both the 
FTSE 250 and FTSE Small Cap indices. The evidence of asymmetry is even more pronounced for the second sub-sample, with an additional significant positive return spillover from the FTSE 250 index to the FTSE Small Cap index. There are marginally significant negative spillovers from the FTSE Small Cap index to the FTSE 250 index in the second sub-sample, and from the FTSE 250 index to the FTSE 100 index in the first sub-sample.

For conditional volatility, the positive spillover effects from the FTSE 100 index to the FTSE 250 index and the FTSE Small Cap index that are present in the full sample are also present in the two sub-samples. The positive spillover from the FTSE 250 index to the FTSE Small Cap index that is present in the full sample is significant for the second sub-sample, but not for the first sub-sample. The positive spillover effect from the FTSE Small Cap index to the FTSE 100 index that is present in the full sample, is marginally significant in the first sub-sample, but absent in the second subsample. However, in the second sub-sample, there is a significantly positive spillover from the FTSE Small Cap index to the FTSE 250 index that is only marginally significant in both the full sample and the first sub-sample. The significant negative spillover from the FTSE 250 index to the FTSE 100 index that is present in the full sample is insignificant in both sub-samples.

The results of this section therefore suggest that the spillover effects in returns and volatility from larger stock portfolios to smaller stock portfolios are robust with respect to the time-period considered. The spillovers in both returns and volatility tend to be stronger in the second sub-sample than the first sub-sample. However, there remain some marginally significant feedback effects in volatility from the portfolios of smaller stocks to the portfolios of larger stocks, but these vary somewhat with the time-period considered. The negative spillover from the FTSE 250 index to the FTSE 100 index is not present in either of the two sub-samples, casting doubt on its robustness and suggesting that it could be, to some extent, spurious.

[Tables 4 and 5] 


\section{The Effect of Non-synchronous Trading on Return and Volatility Spillovers}

Many previous studies (for example, Lo and Mackinlay, 1990a, 1990b) report evidence that non-synchronous trading can potentially induce an asymmetry in the transmission mechanisms of returns between large and small stocks. To investigate the potential effects of non-synchronous trading on our results, we employ the simulation experiments used in Conrad, Gultekin and Kaul (1991) and Kadlec and Patterson (1999). These simulation experiments are based on the non-synchronous trading model first developed by Scholes and Williams (1977) and later generalised by Lo and MacKinlay (1990a). Suppose that the unobservable 'latent' continuously compounded returns $R_{i, t}$ of $N$ securities are generated by the following single-factor model.

$$
R_{i, t}=\alpha_{i}+\beta_{i} M_{t}+\varepsilon_{i, t}, \quad i=1, \ldots, N
$$

where $M_{t} \sim N\left(0, h_{M, t}\right)$ is a zero-mean conditionally heteroscedastic common factor and $\varepsilon_{i, t} \sim N\left(0, h_{\varepsilon}\right)$ is a zero-mean idiosyncratic noise term that is temporally and cross-sectionally independent at all leads and lags. When $M_{t}$ is the return on the market portfolio, equation (7) represents the market model of Sharpe (1964). We assume that the conditional volatility of $M_{t}$ is generated by the following asymmetric GARCH(1,1) model.

$$
h_{M, t}=a_{0}+a_{1} h_{M, t-1}+a_{2} M_{t-1}^{2}+a_{3} I_{t-1} \varepsilon_{M, t-1}^{2}
$$

In order to calibrate this model, we estimate it for the FTSE All Share index for the

full sample period. This yields parameter estimates $\hat{a}_{0}=2.73 * 10^{-6}, \hat{a}_{1}=0.8941$, $\hat{a}_{2}=0.0461$ and $\hat{a}_{3}=0.0668$. Using these parameter estimates, and setting the initial conditional variance, $h_{M, t}$, to the estimated unconditional variance of the FTSE All Share index, we use equation (8) to generate the conditionally heteroscedastic factor return, $M_{t}$. We then use equation (7) to generate the individual stock returns, $R_{i t}$. For all stocks, $\beta_{i}$ is set equal to unity, and $\alpha_{i}$ is set equal to zero. Following Kadlec 
and Patterson (1999), in order to estimate the variance of $\varepsilon_{i, t}$, we first randomly select 100 stocks from the FTSE All Share index at the end of 2002. We regress the daily returns of each of these individual stocks on a constant and the daily return on the FTSE All share index. We then calculate the average variance of the residuals obtained from these regressions and use this as an estimate of $h_{\varepsilon}$.

We now introduce non-synchronous trading into the simulation model. If security $i$ trades in period $t+1$ but did not trade in period $t$, its observed return, $R_{i, t+1}^{o}$, at $t+1$ is simply the sum of its latent returns that period and its latent returns for all past consecutive periods in which it did not trade. Hence, the observed return, $R_{i, t}^{o}$, is given by the following stochastic process.

$$
R_{i, t}^{o}=\sum_{k=0}^{\infty} X_{i, t}(k) R_{i, t-k}, \quad i=1, \ldots, N
$$

where the random weight $X_{i, t}(k)$ is an indicator variable that takes the value 1 when security $i$ trades at time $t$ but has not traded in any of the $k$ previous periods, and takes the value 0 otherwise.

To simulate the observed return, $R_{i, t}^{o}$, we first simulate 4435 daily latent returns, $R_{i, t}$, for 10 large, 10 medium and 10 small stocks. We generate the non-trading history for each individual stock from a Bernoulli process, using the empirical non-trading frequencies of the FTSE 100, the FTSE 250 and the FTSE Small Cap indices. These empirical non-trading frequencies are $0.0008,0.0334$ and 0.0840 , respectively. ${ }^{9}$ Once the latent returns and the non-trading history of large, medium and small stocks are generated, an observed return for each individual stock is computed using equation (9). The daily returns for individual small, medium and large stocks are then aggregated into small, medium, and large portfolios respectively. As a benchmark, we also simulate the return and volatility transmissions under the assumption of no non-

\footnotetext{
${ }^{9}$ These non-trading frequencies are computed from the daily data for the individual stocks that comprise each of the FTSE 100, FTSE 250 and FTSE Small Cap indices between 1 January 1986 and 31 December 2002.
} 
synchronous trading. In this case, we simply repeat the simulation described above, but with the three non-trading probabilities set to zero. The simulations are undertaken using 1000 replications.

To examine the return transmission mechanisms across the three portfolios, we estimate the following regression for each index $i=1,2,3$.

$$
R_{i, t}^{o}=\gamma_{i, 0}+\gamma_{i, 1} R_{1, t-1}^{o}+\gamma_{i, 2} R_{2, t-1}^{o}+\gamma_{i, 3} R_{3, t-1}^{o}+u_{i, t}
$$

where $\gamma_{i, 0}$ is a constant, and $\gamma_{i, 1}, \gamma_{i, 2}$ and $\gamma_{i, 3}$ are the coefficients of the lagged returns of portfolios of the large, medium and small stock portfolios respectively. The volatility transmission mechanisms across all three portfolios are investigated by estimating the following regression.

$$
\hat{u}_{i, t}^{2}=\omega_{i, 0}+\omega_{i, 1} \hat{u}_{1, t-1}^{2}+\omega_{i, 2} \hat{u}_{2, t-1}^{2}+\omega_{i, 3} \hat{u}_{3, t-1}^{2}+\omega_{i, 4} I_{i, t-1} \hat{u}_{i, t-1}^{2}+v_{i, t}
$$

where $\omega_{i, 0}$ is a constant, and $\omega_{i, 1}, \omega_{i, 2}$ and $\omega_{i, 3}$ are the coefficients of the lagged squared return shocks of portfolios of the large, medium and small stock portfolios respectively. These equations are analogous to the multivariate asymmetric GARCH model with spillover effects that is used in the empirical analysis in the previous section.

Table 6 reports the results for the return transmissions. Panel A shows evidence of asymmetric return spillovers from larger stock portfolios to smaller stock portfolios when non-synchronous trading is allowed for in the simulation process, although there is one case that indicates a return spillover from a smaller stock portfolio to a larger stock portfolio. This asymmetry in return spillover effects is consistent with the empirical results reported in the previous section. However, the estimated crossautoregressive parameters in the simulation are considerably lower than those observed in the empirical analysis. When we assume no non-synchronous trading in the simulation process, these spillover effects disappear almost completely. These results therefore suggest that non-synchronous trading may explain a proportion of the asymmetric spillover effects between the FTSE 100, FTSE 250 and FTSE Small Cap indices, but is not able to account for all of it. This is consistent with the findings of 
Lo and MacKinlay (1990a and 1990b), who find that non-synchronous trading, while generating significant return spillovers between large and small stocks, is insufficient to explain the magnitude of lagged cross-correlations in stock returns observed in the US.

[Table 6]

Table 7 suggests that non-synchronous trading is also unlikely to fully explain the observed spillover effects in volatility between the FTSE 100, FTSE 250 and FTSE Small Cap indices. When there is no non-synchronous trading, there is a symmetric pattern of volatility spillovers between the large, medium and small portfolios (which arises from the single facture nature of the simulation model). In contrast with the simulation evidence for return spillovers, the introduction of non-synchronous trading has no distinguishable effect on the pattern of spillovers in volatility. Furthermore, the estimated lagged cross-correlations in squared residuals observed in the simulation with non-synchronous trading are not significantly different from those observed in the simulation with no non-synchronous trading. Thus, it is unlikely that nonsynchronous trading would be able to account for the asymmetric pattern of volatility spillovers that is observed in practice.

[Table 7]

\section{Conclusion}

In this paper, we investigate return and volatility spillover effects between large and small stocks in the UK stock market using the multivariate AR-GJR GARCH-M model. We find that the returns and volatilities of large stocks are important in predicting the future dynamics of smaller stocks, but that the returns and volatilities of smaller stocks have much less impact on the future dynamics of large stocks. Our empirical results suggest that information flow has an influence on the pattern of the transmission mechanisms between large and small stocks. Market-wide information is first incorporated into the prices of large stocks before being impounded into the prices of small stocks. In other words, the prices of small stocks respond with a delay 
to the arrival of market-wide information. Simulation evidence suggests that nonsynchronous trading can account for some of the spillover effects in returns, but not all of it. Similarly, the simulation evidence suggests that non-synchronous trading is unlikely to account for the spillover effects in volatility. The results are consistent with previous studies that find a similar pattern of return and volatility spillovers for the US, and are potentially useful for a range of applications in finance that rely on forecasts of returns and volatilities. 


\section{References}

Aggarwal, R., C. Inclan, and R. Leal (1999), 'Volatility in Emerging Stock Markets', Journal of Financial and Quantitative Analysis, Vol. 34, No. 1, pp. 33-55.

Badrinath, S.G., J.R. Kale, and T.H. Noe (1995), 'Of Shepherds, Sheep and the CrossAutocorrelations in Equity Returns', Review of Financial Studies, Vol. 8, No. 2, pp. 401-430.

Bae, K.-H., and G.A. Karolyi (1994), 'Good News, Bad News and International Spillovers of Stock Return Volatility between Japan and the U.S.', Pacific-Basin Finance Journal, Vol. 2, pp. 405-438.

Bollerslev, T. (1990), 'Modelling the Coherence in Short Run Nominal Exchange Rates: A Multivariate Generalized ARCH Model', Review of Economics and Statistics, Vol. 72, pp. 498-505.

Bollerslev, T., R.Y. Chou, and K.F. Kroner (1992), 'ARCH Modelling in Finance: A Review of Theory and Empirical Evidence', Journal of Econometrics, Vol. 52, pp. 5-59.

Bollerslev, T. and J. Wooldridge (1992), 'Quasi-Maximum Likelihood Estimation and Inference in Dynamic Models with Time-Varying Covariances', Econometric Reviews, Vol. 11, pp. 143-172.

Boudoukh, J., M. Richardson, and R. Whitelaw (1994), 'A Tale of Three Schools: Insights on Autocorrelations of Short-Horizon Returns', Review of Financial Studies, Vol. 7, pp. 539-573.

Campbell, J., A. Lo and A. MacKinlay (1997), The Econometrics of Financial Markets, Princeton University Press, New Jersey.

Chan, K. (1993), 'Imperfect Information and Cross-autocorrelation among Stock Prices' Journal of Finance, Vol. 48, pp. 1211-1230.

Conrad, J., M. Gultekin, and G. Kaul (1991), 'Asymmetric Predictability of Conditional Variances', Review of Financial Studies, Vol. 4, pp. 597-622.

Draper, P., and K. Paudyal (1997), 'Microstructure and Seasonality in the UK Equity Market', Journal of Business Finance and Accounting, Vol. 24, pp. 1177-1204.

Engle, R.F., and K.F. Kroner (1995), 'Multivariate Simultaneous Generalized ARCH', Econometric Theory, Vol. 11, No. 1, pp.122-150.

Engle, R.F., and V.K. Ng (1993), 'Measuring and Testing the Impact of News on Volatility', Journal of Finance, Vo. 48, No. 5, pp. 1749-1778

Glosten, L., R. Jagannathan, and D. Runkle (1993), 'Season Patterns in the Volatility of Stock Index Excess Returns’, Journal of Finance, Vol. 48, pp. 1779-1801. 
Grinblatt, M., S. Titman, and R. Wermers (1995), 'Momentum Investment Strategies, Portfolio Performance, and Herding: A Study of Mutual Fund Behavior', American Economic Review, Vol. 85, pp. 1088-1105.

Hamao, Y., R.W. Masulis, and V.K. Ng (1990), 'Correlations in Price Changes and Volatility across International Stock Markets', The Review of Financial Studies, Vol. 3, No. 2, pp. 281-307.

Hasan, I., and B.B. Francis (1998), 'Macroeconomic Factors and the Asymmetric Predictability of Conditional Variances', European Financial Management, Vol. 4, No. 2, pp. 207-230.

Hung, M.W., C.F. Lee, and L.C. So (2003), 'The Impact of Foreign-listed Single Stock Futures on the Domestic Underlying Stock Markets', Applied Economics Letters, Vol. 10, No.9, pp. 567-574.

Kadlec, G.B., and D.M. Patterson (1999), 'A Transactions Data Analysis of Nonsynchronous Trading', The Review of Financial Studies, Vol. 12, No. 3, pp. 609-630.

Keim, D., and A. Madhavan (1995), 'Anatomy of the Trading Process: Empirical Evidence on the Behavior of Institutional Traders', Journal of Financial Economics, Vol. 37, pp. 371-398.

Kroner, K., and V.K. Ng (1998), 'Modeling Asymmetric Comovement of Asset Returns', Review of Financial Studies, Vol. 11, No. 4.

Lakonishok, J., and S. Smidt (1988), 'Are Seasonal Anomalies Real?: A Ninety Year Perspective', Review of Financial Studies, Vol. 1, pp. 403-425.

Lo, A. W., and A. C. MacKinlay (1990a), 'An Econometric Analysis of Nonsynchronous Trading', Journal of Econometrics, Vol. 45, pp. 181-211.

Lo, A. W., and A. C. MacKinlay (1990b), 'When are Constrarian Profits due to Shock Market Overreaction?', Review of Financial Studies, Vol. 3, pp. 175-205.

McQueen, G., M. Pinegar, and S. Thorley (1996), 'Delayed Reaction to Good News and the Cross-Autocorrelation of Portfolio Returns', Journal of Finance, Vol. 51, No. 3, pp. 889-919.

Mech, T. (1993), 'Portfolio Autocorrelation', Journal of Financial Economics, Vol. 34, pp. 307-44.

Ng, V.K., R.P. Chang and R. Chou (1991), 'An Examination of the Behavior of International Stock Market Volatility, in: S. Ghon Rhee and Rosita P. Chang, eds.', Pacific-Basin Capital Market Research, Vol. 2 (North-Holland, Amsterdam), Pp. 245-260.

Roll, R. (1988), 'The International Crash of October 1987', Financial Analysts Journal, September-October, pp. 19-35. 
Ross, S.A. (1989), 'Information and Volatility: The No-Arbitrage Martingale Approach to Timing and Resolution Irrelevancy', Journal of Finance, Vol. 44, pp. 117.

Scholes, M., and J. Williams (1977), 'Estimating Betas from Nonsynchronous Data', Journal of Financial Economics, Vol. 5, pp. 309-327.

Schwert, G.W. (1990), 'Stock Volatility and the Crash of '87', The Review of Financial Studies, Vol. 3, No. 1, pp. 77-102.

Sharpe, W.F. (Sept. 1964), 'Capital Asset Prices: A Theory of Market Equilibrium under Conditions of Risk', Journal of Finance, pp. 425-442.

Thedossiou, P. and U. Lee (1993), 'Mean and Volatility Spillovers across Major National Stock Markets: Further Empirical Evidence', Journal of Financial Research, Vol. 16, No. 4, pp. 337-350.

Tinic, S. M. and R.R. West (1984), 'Risk and Return: January vs the Rest of the Year', Journal of Financial Economics, Vol. 13, pp. 561-74.

Wang, S.S., O.M. Rui, and M. Firth (2002), 'Return and Volatility Behavior of Dually-traded Stocks: the Case of Hong Kong', Journal of International Money and Finance, Vol. 21, pp.265-293.

White, H., 1982, "Maximum Likelihood Estimation of Misspecified Models", Econometrica 50, 1-25. 
Table 1 Descriptive Statistics and Autocorrelations

\section{Panel A: Descriptive Statistics}

$\begin{array}{lccccc}\text { Index } & \text { Mean } & \text { St Dev } & \text { Skewness } & \text { Kurtosis } & \text { JB Statistic } \\ \text { FTSE 100 } & 0.0002 & 0.0106 & -0.85 & 11.17 & 22436^{* *} \\ \text { FTSE 250 } & 0.0003 & 0.0078 & -1.93 & 26.79 & 131241^{* *} \\ \text { FTSE SC } & 0.0002 & 0.0064 & -3.25 & 42.11 & 325235^{* *}\end{array}$

\section{Panel B: Autocorrelations}

\begin{tabular}{|c|c|c|c|c|c|}
\hline \multicolumn{3}{|c|}{$R_{t}$} & \\
\hline FTSE 100 & FTSE 250 & FTSE SC & FTSE 100 & FTSE 250 & FTSE SC \\
\hline $0.05 * *$ & $0.22 * *$ & $0.30 * *$ & $0.51 * *$ & $0.35 * *$ & $0.29 * *$ \\
\hline$-0.04 * *$ & $0.12 * *$ & $0.20 * *$ & $0.29 * *$ & $0.21 * *$ & $0.16^{* *}$ \\
\hline$-0.04 * *$ & $0.08 * *$ & $0.18 * *$ & $0.18 * *$ & $0.14 * *$ & $0.12 * *$ \\
\hline $0.04 * *$ & $0.14 * *$ & $0.20 * *$ & $0.18 * *$ & $0.34 * *$ & $0.25 * *$ \\
\hline $30.18^{* *}$ & $391.87 * *$ & $913.32 * *$ & $1,791.39 * *$ & $1,335.64 * *$ & $834.71 * *$ \\
\hline
\end{tabular}

Notes: Panel A reports the mean, standard deviation and skewness and excess kurtosis coefficients, and the Jarque-Bera test statistic for normality. Panel B reports the first four autocorrelations for the returns and squared returns of each index and fourth-order Ljung-Box statistics. '*' and '**' denote statistical significance at the $5 \%$ level and the $1 \%$ level respectively. 
Table 2 The Multivariate AR(4)-GJR GARCH(1,1)-M Model

\begin{tabular}{|c|c|c|c|c|c|c|}
\hline & \multicolumn{2}{|c|}{ FTSE 100} & \multicolumn{2}{|c|}{ FTSE 250} & \multicolumn{2}{|c|}{ FTSE Small Cap } \\
\hline & Coeff & t-stat & Coeff & t-stat & Coeff & t-stat \\
\hline$\alpha_{i, 0}$ & -0.0005 & -1.15 & -0.0002 & -0.72 & -0.0002 & -1.75 \\
\hline$\alpha_{i, 1}$ & -0.0750 & $-6.22 * *$ & 0.1304 & $11.56^{* *}$ & 0.3437 & $22.04 * *$ \\
\hline$\alpha_{i, 2}$ & -0.0061 & -0.53 & 0.0763 & $6.76^{* *}$ & 0.8640 & $4.94 * *$ \\
\hline$\alpha_{i, 3}$ & -0.0321 & $-2.86^{* *}$ & 0.0188 & 1.75 & 0.0664 & $4.65^{* *}$ \\
\hline$\alpha_{i, 4}$ & 0.0030 & 0.27 & 0.0474 & $4.84^{* *}$ & 0.0725 & $5.93^{* *}$ \\
\hline$\gamma_{i}$ & 0.1317 & $2.46^{*}$ & 0.1225 & $2.57^{*}$ & 0.1169 & $3.51^{* *}$ \\
\hline$c_{i, 1}$ & -0.0513 & $-3.72 * *$ & -0.0424 & $-4.77 * *$ & -0.0290 & $-4.20 * *$ \\
\hline$c_{i, 2}$ & -0.0277 & $-5.01 * *$ & -0.0214 & $-2.99 * *$ & -0.0153 & $-2.45^{*}$ \\
\hline$c_{i, 3}$ & -0.0012 & -0.80 & 0.0012 & 1.47 & 0.0009 & 1.42 \\
\hline$c_{i, 4}$ & -0.0007 & $-2.87 * *$ & -0.0008 & $-4.66^{* *}$ & -0.0003 & -1.90 \\
\hline$\beta_{i, 0} \times 10^{2}$ & 0.0004 & $3.75^{* *}$ & 0.0004 & $5.16^{* *}$ & 0.0001 & $2.31^{*}$ \\
\hline$\beta_{i, 1}$ & 0.8935 & $57.47^{* *}$ & 0.7973 & $25.58^{* *}$ & 0.7353 & $19.75^{* *}$ \\
\hline$\beta_{i, 2}$ & 0.0523 & $5.23 * *$ & 0.1219 & $4.85^{* *}$ & 0.1862 & $5.19^{* *}$ \\
\hline$\lambda_{i}$ & 0.0431 & $4.13^{* *}$ & 0.0242 & 1.26 & 0.0778 & 1.49 \\
\hline$d_{i, 1} \times 10^{2}$ & 0.0155 & 1.59 & 0.0134 & $1.99^{*}$ & 0.0129 & $2.01 *$ \\
\hline$d_{i, 2} \times 10^{2}$ & 0.0081 & 1.63 & 0.0088 & 1.70 & 0.0065 & 1.68 \\
\hline$d_{i, 3} \times 10^{2}$ & 0.0013 & 1.75 & 0.0006 & $2.63^{* *}$ & 0.0004 & 1.69 \\
\hline$d_{i, 4} \times 10^{2}$ & -0.0008 & -1.61 & -0.0005 & $-2.34 *$ & 0.0001 & 0.66 \\
\hline$\rho_{F T S E 100, F T S E 250}$ & 0.7981 & $131.89 * *$ & & & & \\
\hline$\rho_{\text {FTSE } 100, F T S E S C}$ & 0.6770 & $76.45^{* *}$ & & & & \\
\hline$\rho_{\text {FTSE25, FTSESC }}$ & 0.8444 & $124.61 * *$ & & & & \\
\hline $\mathrm{LB}(4)$ & 76.7422 & {$[0.00]$} & 62.3303 & {$[0.00]$} & 18.0303 & {$[0.00]$} \\
\hline $\mathrm{LB}^{2}(4)$ & 1.5436 & {$[0.82]$} & 2.5457 & {$[0.64]$} & 0.9340 & {$[0.92]$} \\
\hline
\end{tabular}

Notes: The table reports the estimation for the multivariate AR(4)-GJR GARCH(1,1)-M model given by

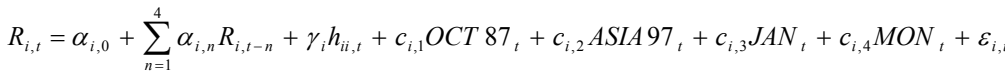

$$
\begin{aligned}
& h_{i i, t}=\beta_{i, 0}+\beta_{i, 1} h_{i i, t-1}+\beta_{i, 2} \varepsilon_{i, t-1}^{2}+\lambda_{i} I_{i, t-1} \varepsilon_{i, t-1}^{2}+d_{i, 1} O C T 87_{t}+d_{i, 2} \text { ASIA97 }_{t}+d_{i, 3} J_{A N}+d_{i, 4} \text { MON }_{t} \\
& h_{i j, t}=\rho_{i j}\left(\sqrt{h_{i i, t}} \sqrt{h_{j j, t}}\right)
\end{aligned}
$$

t-statistics are in parenthesis. '*' and '**' denote statistical significance at the $5 \%$ level and the $1 \%$ level respectively. $\mathrm{LB}(4)$ and $\mathrm{LB}^{2}(4)$ are the fourth-order Ljung-Box statistics for standardized residuals and squared standardized residuals, respectively. The p-values of these statistics are reported in parentheses. 
Table 3 The Multivariate AR(4)-GJR GARCH(1,1)-M Model with Spillovers

\begin{tabular}{|c|c|c|c|c|c|c|}
\hline & \multicolumn{2}{|c|}{ FTSE 100} & \multicolumn{2}{|c|}{ FTSE 250} & \multicolumn{2}{|c|}{ FTSE Small Cap } \\
\hline & Coeff & t-stat & Coeff & t-stat & Coeff & t-stat \\
\hline$\alpha_{i, 0}$ & -0.0008 & -1.80 & -0.0004 & -1.78 & -0.0003 & $-2.52 *$ \\
\hline$\alpha_{i, 1}$ & 0.0421 & 1.46 & 0.1340 & $4.51^{* *}$ & 0.2191 & $7.41^{* *}$ \\
\hline$\alpha_{i, 2}$ & -0.0132 & -0.94 & 0.0700 & $5.68 * *$ & 0.1070 & $5.92 * *$ \\
\hline$\alpha_{i, 3}$ & -0.0289 & $-2.07 *$ & 0.0285 & $2.52 *$ & 0.0853 & $7.03 * *$ \\
\hline$\alpha_{i, 4}$ & 0.0018 & 0.15 & 0.0504 & $5.41 * *$ & 0.0831 & $7.22 * *$ \\
\hline$\gamma_{i}$ & 0.1670 & $3.50 * *$ & 0.1641 & $4.08^{* *}$ & 0.1371 & $4.35^{* *}$ \\
\hline$c_{i, 1}$ & -0.0485 & $-3.48^{* *}$ & -0.0450 & $-4.43 * *$ & -0.0294 & $-3.85^{* *}$ \\
\hline$c_{i, 2}$ & -0.0251 & $-5.40 * *$ & -0.0197 & $-3.08^{* *}$ & -0.0138 & $-2.50^{*}$ \\
\hline$c_{i, 3}$ & -0.0010 & -0.60 & 0.0012 & 1.46 & 0.0011 & 1.85 \\
\hline$c_{i, 4}$ & -0.0005 & -1.60 & -0.0007 & $-3.14 * *$ & -0.0002 & -1.27 \\
\hline$w_{i, F T S E 100}$ & - & - & 0.0876 & $5.20 * *$ & 0.0830 & $7.81^{* *}$ \\
\hline$w_{i, F T S E 250}$ & -0.0412 & -0.79 & - & - & 0.0340 & 1.60 \\
\hline$w_{i, F T S E S C}$ & 0.0060 & 0.10 & -0.0417 & -1.17 & - & - \\
\hline$\beta_{i, 0} \times 10^{2}$ & 0.0000 & $3.93 * *$ & 0.0000 & $5.25^{* *}$ & 0.0000 & 1.50 \\
\hline$\beta_{i, 1}$ & 0.8886 & $50.94 * *$ & 0.7360 & $21.40^{* *}$ & 0.7012 & $30.20 * *$ \\
\hline$\beta_{i, 2}$ & 0.0827 & $6.27 * *$ & 0.1055 & $4.58^{* *}$ & 0.1565 & $5.75^{* *}$ \\
\hline$\lambda_{i}$ & 0.0334 & $2.93 * *$ & -0.0073 & -0.36 & 0.0754 & 1.26 \\
\hline$d_{i, 1} \times 10^{2}$ & 0.0002 & 1.63 & 0.0001 & 1.71 & 0.0001 & 1.73 \\
\hline$d_{i, 2} \times 10^{2}$ & 0.0001 & $2.37 *$ & 0.0001 & $2.03^{*}$ & 0.0001 & 1.92 \\
\hline$d_{i, 3} \times 10^{2}$ & 0.0000 & 1.44 & 0.0000 & $2.29^{*}$ & 0.0000 & 1.84 \\
\hline$d_{i, 4} \times 10^{2}$ & -0.0000 & $-2.21 *$ & -0.0000 & $-2.45^{*}$ & 0.0000 & 0.54 \\
\hline$z_{i, F T S E 100}$ & - & - & 0.0318 & $4.72 * *$ & 0.0103 & $2.59 * *$ \\
\hline$z_{i, F T S E 250}$ & -0.0388 & $-4.85 * *$ & - & - & 0.0247 & $2.18^{*}$ \\
\hline$z_{i, F T S E S C}$ & 0.0140 & $2.26^{*}$ & 0.0646 & 1.65 & - & - \\
\hline$\rho_{F T S E 100, F T S E 250}$ & 0.8004 & $135.04 * *$ & & & & \\
\hline$\rho_{F T S E 100, F T S E S C}$ & 0.6783 & $74.70^{* *}$ & & & & \\
\hline$\rho_{F T S E 250, F T S E S C}$ & 0.8462 & $137.22 * *$ & & & & \\
\hline $\mathrm{LB}(4)$ & 10.5768 & {$[0.03]$} & 15.2614 & {$[0.00]$} & 6.4418 & {$[0.17]$} \\
\hline $\mathrm{LB}^{2}(4)$ & 1.9586 & {$[0.74]$} & 1.2802 & {$[0.87]$} & 1.1745 & {$[0.88]$} \\
\hline
\end{tabular}

Notes: The table reports the estimation for the multivariate AR(4)-GJR GARCH(1,1)-M model with spillover effects, given by

$$
\begin{aligned}
& R_{i, t}=\alpha_{i, 0}+\sum_{n=1}^{4} \alpha_{i, n} R_{i, t-n}+\gamma_{i} h_{i i, t}+c_{i, 1} \text { OCT } 87_{t}+c_{i, 2} \text { ASIA } 97_{t}+c_{i, 3} J A N_{t}+c_{i, 4} M O N_{t}+\sum_{j=1, j \neq i}^{3} w_{i, j} R_{j, t-1}+\varepsilon_{i, t} \\
& h_{i i, t}=\beta_{i, 0}+\beta_{i, 1} h_{i, t-1}+\beta_{i, 2} \varepsilon_{i, t-1}^{2}+\lambda_{i} I_{i, t-1} \varepsilon_{i, t-1}^{2}+d_{i, 1} O C T 87_{t}+d_{i, 2} \operatorname{ASIAS7}_{t}+d_{i, 3} J A N_{t}+d_{i, 4} M O N_{t}+\sum_{j=1, j \neq j}^{3} z_{i, j} \varepsilon_{j, t-1}^{2} \\
& h_{i j, t}=\rho_{i j}\left(\sqrt{h_{i i, t}} \sqrt{h_{j j, t}}\right)
\end{aligned}
$$

t-statistics are in parenthesis. ' $*$ ' and ' $* *$ ' denote statistical significance at the $5 \%$ level and the $1 \%$ level respectively. $\mathrm{LB}(4)$ and $\mathrm{LB}^{2}(4)$ are the fourth-order Ljung-Box statistics for standardized residuals and squared standardized residuals, respectively. The p-values of these statistics are reported in parentheses. 
Table 4 The Multivariate AR(4)-GJR GARCH(1,1)-M Model with Spillovers over the period: $1^{\text {st }}$ Jan 1986 to $30^{\text {th }}$ Jun 1994

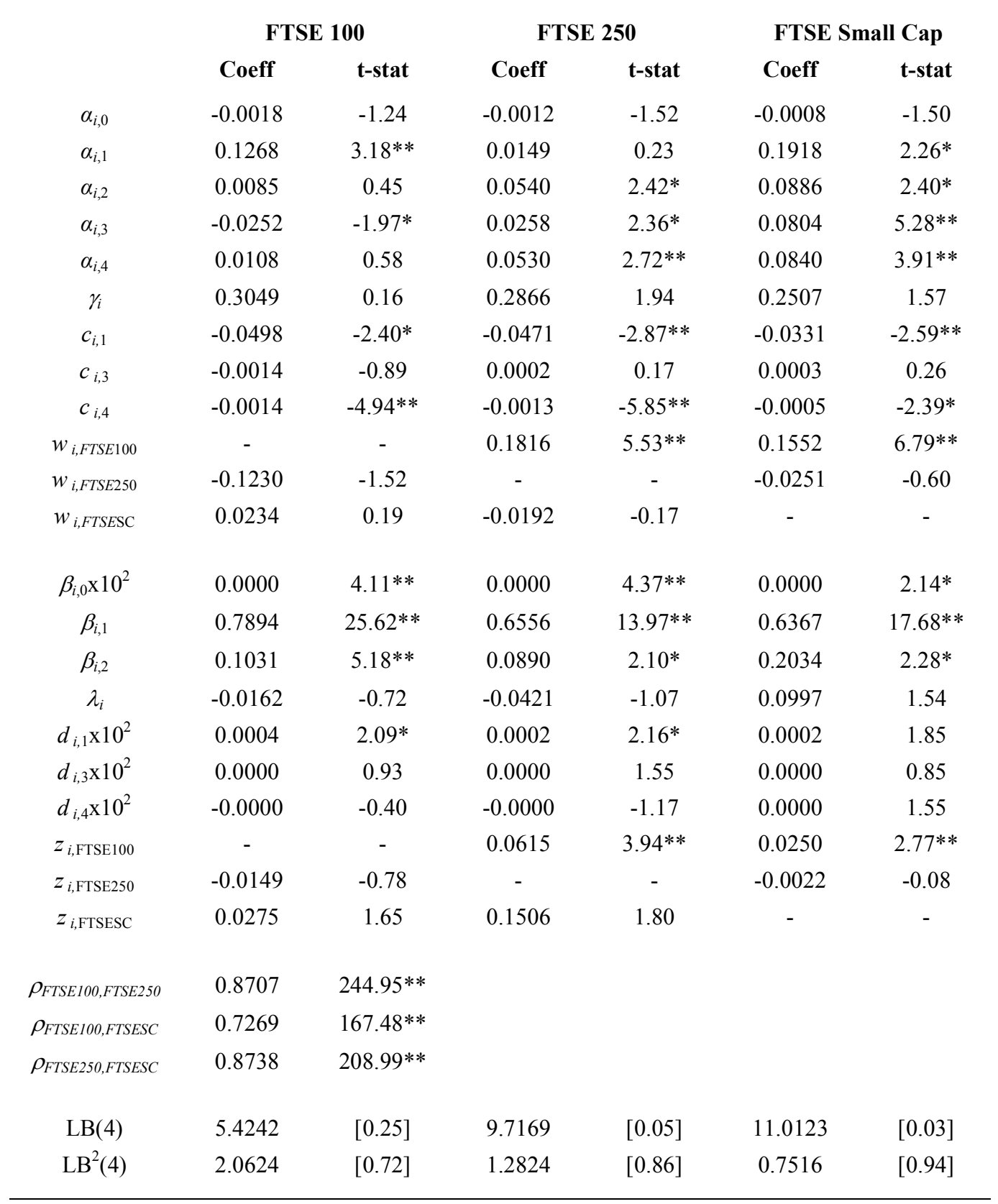

Notes: The table reports the estimation for the multivariate AR(4)-GJR GARCH(1,1)-M model with spillover effects, given by

$$
\begin{aligned}
& R_{i, t}=\alpha_{i, 0}+\sum_{n=1}^{4} \alpha_{i, n} R_{i, t-n}+\gamma_{i} h_{i i, t}+c_{i, 1} \text { OCT } 87_{t}+c_{i, 3} J A N_{t}+c_{i, 4} M O N_{t}+\sum_{j=1, j \neq i}^{3} w_{i, j} R_{j, t-1}+\varepsilon_{i, t} \\
& h_{i i, t}=\beta_{i, 0}+\beta_{i, 1} h_{i i, t-1}+\beta_{i, 2} \varepsilon_{i, t-1}^{2}+\lambda_{i} I_{i, t-1} \varepsilon_{i, t-1}^{2}+d_{i, 1} O C T 87_{t}+d_{i, 3} J A N_{t}+d_{i, 4} M O N_{t}+\sum_{j=1, j \neq i}^{3} z_{i, j} \varepsilon_{j, t-1}^{2} \\
& h_{i j, t}=\rho_{i j}\left(\sqrt{h_{i i, t}} \sqrt{h_{j j, t}}\right)
\end{aligned}
$$

$\mathrm{t}$-statistics are in parenthesis. ' $*$ ' and ' $* *$ ' denote statistical significance at the $5 \%$ level and the $1 \%$ level respectively. $\mathrm{LB}(4)$ and $\mathrm{LB}^{2}(4)$ are the fourth-order Ljung-Box statistics for standardized residuals and squared standardized residuals, respectively. The p-values of these statistics are reported in parentheses. 
Table 5 The Multivariate AR(4)-GJR GARCH(1,1)-M Model with Spillovers over the period: $1^{\text {st }}$ Jul 1994 to $31^{\text {st }}$ Dec 2002

\begin{tabular}{|c|c|c|c|c|c|c|}
\hline & \multicolumn{2}{|c|}{ FTSE 100} & \multicolumn{2}{|c|}{ FTSE 250} & \multicolumn{2}{|c|}{ FTSE Small Cap } \\
\hline & Coeff & t-stat & Coeff & t-stat & Coeff & t-stat \\
\hline$\alpha_{i, 0}$ & -0.0004 & -0.79 & -0.0002 & -0.72 & -0.0002 & $-2.04 *$ \\
\hline$\alpha_{i, 1}$ & 0.0433 & $2.75^{* *}$ & 0.1725 & $5.83^{* *}$ & 0.2430 & $8.60 * *$ \\
\hline$\alpha_{i, 2}$ & -0.0378 & $-3.56^{* *}$ & 0.0823 & $8.34^{* *}$ & 0.1103 & $10.46^{* *}$ \\
\hline$\alpha_{i, 3}$ & -0.0403 & $-2.86^{* *}$ & 0.0251 & $2.15^{*}$ & 0.0920 & $6.65^{* *}$ \\
\hline$\alpha_{i, 4}$ & -0.0042 & -0.47 & 0.0446 & $4.64 * *$ & 0.0754 & $5.87 * *$ \\
\hline$\gamma_{i}$ & 0.1070 & 1.94 & 0.1223 & $2.30^{*}$ & 0.1235 & $3.53^{* *}$ \\
\hline$c_{i, 2}$ & -0.0236 & $-6.07 * *$ & -0.0186 & $-3.10^{* *}$ & -0.0135 & $-2.25 *$ \\
\hline$c_{i, 3}$ & -0.0013 & -0.71 & 0.0011 & 1.85 & 0.0012 & $2.18^{*}$ \\
\hline$c_{i, 4}$ & 0.0005 & 1.71 & -0.0002 & -1.87 & -0.0001 & -1.35 \\
\hline$w_{i, F T S E 100}$ & - & - & 0.0721 & $9.94 * *$ & 0.0633 & $11.53 * *$ \\
\hline$w_{i, F T S E 250}$ & -0.0408 & -0.86 & - & - & 0.0432 & $2.25^{*}$ \\
\hline$w_{i, F T S E S C}$ & -0.0930 & -1.60 & -0.0765 & -1.92 & - & - \\
\hline$\beta_{i, 0} \times 10^{2}$ & 0.0000 & $3.69^{* *}$ & 0.0000 & $4.82 * *$ & 0.0000 & $2.11^{*}$ \\
\hline$\beta_{i, 1}$ & 0.9002 & $29.79^{* *}$ & 0.7455 & $22.77^{* *}$ & 0.7180 & $31.24 * *$ \\
\hline$\beta_{i, 2}$ & 0.0455 & $2.82 * *$ & 0.0875 & $3.94 * *$ & 0.1448 & $8.57 * *$ \\
\hline$\lambda_{i}$ & 0.0550 & $3.05^{* *}$ & 0.0117 & 0.53 & 0.0839 & $3.28^{* *}$ \\
\hline$d_{i, 2} \times 10^{2}$ & 0.0000 & 1.04 & 0.0000 & $3.48^{* *}$ & 0.0000 & $2.78 * *$ \\
\hline$d_{i, 3} \times 10^{2}$ & 0.0000 & 1.20 & 0.0000 & 0.99 & 0.0000 & 1.36 \\
\hline$d_{i, 4 \times 10^{2}}$ & -0.0000 & $-3.19 * *$ & -0.0000 & $-2.87^{* *}$ & -0.0000 & -0.88 \\
\hline$z_{i, \mathrm{FTSE} 100}$ & - & - & 0.0228 & $3.75^{* *}$ & 0.0053 & $2.52^{*}$ \\
\hline$z_{i, \mathrm{FTSE} 250}$ & 0.0071 & 0.27 & - & - & 0.0237 & $2.61 * *$ \\
\hline$z_{i, \mathrm{FTSESC}}$ & 0.0072 & 0.22 & 0.0664 & $2.45^{*}$ & - & - \\
\hline$\rho_{F T S E I 00, F T S E 250}$ & 0.7284 & $77.94 * *$ & & & & \\
\hline$\rho_{\text {FTSE } 100, F T S E S C}$ & 0.6350 & $60.47^{* *}$ & & & & \\
\hline$\rho_{F T S E 250, F T S E S C}$ & 0.8116 & $115.22 * *$ & & & & \\
\hline $\mathrm{LB}(4)$ & 6.2476 & {$[0.18]$} & 11.8394 & {$[0.02]$} & 4.0297 & [0.40] \\
\hline $\mathrm{LB}^{2}(4)$ & 3.2103 & {$[0.52]$} & 0.5085 & {$[0.97]$} & 3.0852 & {$[0.54]$} \\
\hline
\end{tabular}

Notes: The table reports the estimation for the multivariate AR(4)-GJR GARCH(1,1)-M model with spillover effects, given by

$$
\begin{aligned}
& R_{i, t}=\alpha_{i, 0}+\sum_{n=1}^{4} \alpha_{i, n} R_{i, t-n}+\gamma_{i} h_{i i, t}+c_{i, 2} A S I A 97_{t}+c_{i, 3} J A N_{t}+c_{i, 4} M O N_{t}+\sum_{j=1, j \neq i}^{3} w_{i, j} R_{j, t-1}+\varepsilon_{i, t} \\
& h_{i i, t}=\beta_{i, 0}+\beta_{i, 1} h_{i, t,-1}+\beta_{i, 2} \varepsilon_{i, t-1}^{2}+\lambda_{i} I_{i, t-1} \varepsilon_{i, t-1}^{2}+d_{i, 2} A S I A 97_{t}+d_{i, 3} J A N_{t}+d_{i, 4} M O N_{t}+\sum_{j=1, j \neq i}^{3} z_{i, j} \varepsilon_{j, t-1}^{2} \\
& h_{i j, t}=\rho_{i j}\left(\sqrt{h_{i i, t}} \sqrt{h_{j j, t}}\right)
\end{aligned}
$$

t-statistics are in parenthesis. ' $*$ ' and ' $* *$ ' denote statistical significance at the $5 \%$ level and the $1 \%$ level respectively. $\mathrm{LB}(4)$ and $\mathrm{LB}^{2}(4)$ are the fourth-order Ljung-Box statistics for standardized residuals and squared standardized residuals, respectively. The p-values of these statistics are reported in parentheses. 


\section{Table 6 Simulation Results for Non-Synchronous trading: Returns}

\section{Panel A: Non-synchronous Trading}

$\begin{array}{lccc} & R_{1, t}^{o} & R_{2, t}^{o} & R_{3, t}^{o} \\ R_{1, t-1}^{o} & & & \\ R_{2, t-1}^{o} & 0.0010 & 0.0158 & 0.0270 \\ R_{3, t-1}^{o} & (0.81) & (14.49)^{* *} & (19.80)^{* *} \\ & -0.0014 & 0.0063 & 0.0399 \\ & (-1.02) & (5.30)^{* *} & (26.18)^{* *} \\ & -0.0011 & 0.0033 & -0.0010 \\ & (-1.43) & (4.64)^{* *} & (-1.12)\end{array}$

Panel B: Synchronous Trading

$\begin{array}{lccc} & R_{1, t}^{o} & R_{2, t}^{o} & R_{3, t}^{o} \\ R_{1, t-1}^{o} & & & \\ R_{2, t-1}^{o} & -0.0001 & 0.0007 & 0.0010 \\ R_{3, t-1}^{o} & (-0.12) & (0.64) & (0.73) \\ & 0.0001 & -0.0005 & -0.0013 \\ & (0.08) & (-0.41) & (-0.86) \\ & 0.0003 & 0.0001 & -0.0005 \\ & (0.47) & (0.18) & (-0.51)\end{array}$

Notes: $R_{1, t}^{o}, R_{2, t}^{o}$ and $R_{3, t}^{o}$ are the simulated observed returns of large, medium and small stock portfolios respectively. The table reports the average coefficients in the regression $R_{i, t}^{o}=\gamma_{i, 0}+\gamma_{i, 1} R_{1, t-1}^{o}+\gamma_{i, 2} R_{2, t-1}^{o}+\gamma_{i, 3} R_{3, t-1}^{o}+u_{i, t}$ for each index $i=1,2,3$. For the non-synchronous trading case, the non-trading probabilities are set to the empirical non-trading frequencies of $0.0008,0.0334$ and 0.0840 . For the synchronous trading case, the non-trading probabilities are set equal to zero. The simulation is based on 1000 replications. t-statistics are reported in parentheses. '*' and '**' denote statistical significance at the $5 \%$ level and the $1 \%$ level respectively. 


\section{Panel A: Non-synchronous Trading}

$\begin{array}{lccc} & \hat{u}_{1, t}^{2} & \hat{u}_{2, t}^{2} & \hat{u}_{3, t}^{2} \\ & & & \\ \hat{u}_{1, t-1}^{2} & 0.0517 & 0.0513 & 0.0480 \\ \hat{u}_{2, t-1}^{2} & (31.31)^{* *} & (34.88)^{* *} & (25.96)^{* *} \\ \hat{u}_{3, t-1}^{2} & 0.0765 & 0.0692 & 0.0699 \\ & (42.67)^{* *} & (40.94)^{* *} & (34.44)^{* *} \\ & 0.0137 & 0.0127 & 0.0054 \\ & (15.52)^{* *} & (15.68)^{* *} & (5.41)^{* *}\end{array}$

\section{Panel B: Synchronous Trading}

$\begin{array}{lccc} & \hat{u}_{1, t}^{2} & \hat{u}_{2, t}^{2} & \hat{u}_{3, t}^{2} \\ & & & \\ \hat{u}_{1, t-1}^{2} & 0.0470 & 0.0472 & 0.0466 \\ \hat{u}_{2, t-1}^{2} & (31.81)^{* *} & (32.99)^{* *} & (25.82)^{* *} \\ \hat{u}_{3, t-1}^{2} & 0.0757 & 0.0761 & 0.0749 \\ & (42.06)^{* *} & (43.41)^{* *} & (34.05)^{* *} \\ & 0.0124 & 0.0121 & 0.0121 \\ & (15.79)^{* *} & (16.20)^{* *} & (11.55)^{* *}\end{array}$

Notes: $\hat{u}_{1, t}^{2}, \hat{u}_{2, t}^{2}$ and $\hat{u}_{3, t}^{2}$ are the simulated squared residuals of large, medium and small stock portfolios respectively. The table reports the average coefficients in the regression $\hat{u}_{i, t}^{2}=\omega_{i, 0}+\omega_{i, 1} \hat{u}_{1, t-1}^{2}+\omega_{i, 2} \hat{u}_{2, t-1}^{2}+\omega_{i, 3} \hat{u}_{3, t-1}^{2}+\omega_{i, 4} I_{t-1} \hat{u}_{i, t-1}^{2}+v_{i, t}$ for each index $i=1,2,3$. For the nonsynchronous trading case, the non-trading probabilities are set to the empirical non-trading frequencies of $0.0008,0.0334$ and 0.0840 . For the synchronous trading case, the non-trading probabilities are set equal to zero. The simulation is based on 1000 replications. t-statistics are reported in parentheses. '*'and '**' denote statistical significance at the $5 \%$ level and the $1 \%$ level respectively. 